\title{
Three-dimensional regional strain analysis in porcine myocardial infarction: a 3T magnetic resonance tagging study
}

Sahar Soleimanifard ${ }^{1}$, Khaled Z Abd-Elmoniem², Tetsuo Sasano ${ }^{3}$, Harsh K Agarwal ${ }^{4}$, M Roselle Abraham $^{3}$, Theodore P Abraham ${ }^{3}$ and Jerry L Prince ${ }^{1,5^{*}}$

\begin{abstract}
Background: Previous studies of mechanical strain anomalies in myocardial infarction (MI) have been largely limited to analysis of one-dimensional (1D) and two-dimensional (2D) strain parameters. Advances in cardiovascular magnetic resonance (CMR) methods now permit a complete three-dimensional (3D) interrogation of myocardial regional strain. The aim of this study was to investigate the incremental value of CMR-based 3D strain and to test the hypothesis that 3D strain is superior to 1D or 2D strain analysis in the assessment of viability using a porcine model of infarction.
\end{abstract}

Methods: Infarction was induced surgically in 20 farm pigs. Cine, late gadolinium enhancement, and CMR tagging images were acquired at 11 days before (baseline), and 11 days (early) and 1 month (late) after induction of infarct. Harmonic phase analysis was performed to measure circumferential, longitudinal, and radial strains in myocardial segments, which were defined based on the transmurality of delayed enhancement. Univariate, bivariate, and multivariate logistic regression models of strain parameters were created and analyzed to compare the overall diagnostic accuracy of 3D strain analysis with $1 \mathrm{D}$ and 2D analyses in identifying the infarct and its adjacent regions from healthy myocardium.

Results: 3D strain differed significantly in infarct, adjacent, and remote segments $(p<0.05)$ at early and late post-MI. In univariate, bivariate, and multivariate analyses, circumferential, longitudinal, and radial strains were significant factors $(p<0.001)$ in differentiation of infarct and adjacent segments from baseline values. In identification of adjacent segments, receiver operating characteristic analysis using the 3D strain multivariate model demonstrated a significant improvement $(p<0.01)$ in overall diagnostic accuracy in comparison with 2D (circumferential and radial) and 1D (circumferential) models (3D: 96\%, 2D: 81\%, and 1D: 71\%). A similar trend was observed in identification of infarct segments.

Conclusions: Cumulative 3D strain information accurately identifies infarcts and their neighboring regions from healthy myocardium. The 3D interrogation of myocardial contractility provides incremental diagnostic accuracy in delineating the dysfunctional and nonviable myocardium in comparison with 1D or 2D quantification of strain. The infarct neighboring regions are the major beneficiaries of the 3D assessment of regional strain.

Keywords: Magnetic resonance tagging, Harmonic phase analysis, Three-dimensional regional strain, Myocardial infarction, Diagnostic accuracy

\footnotetext{
* Correspondence: prince@jhu.edu

${ }^{1}$ Department of Electrical and Computer Engineering, Johns Hopkins

University, 3400 N. Charles Street, Clark Hall 201, Baltimore, MD 21218, USA

${ }^{5}$ Department of Radiology and Radiological Science, Johns Hopkins

University, 3400 N. Charles Street, Clark Hall 201, Baltimore, MD 21218, USA

Full list of author information is available at the end of the article
} 


\section{Background}

Cardiovascular magnetic resonance (CMR) is the gold standard for the assessment of regional myocardial strain, which provides a sensitive and quantitative indicator of myocardial function and viability $[1,2]$. Several CMR techniques including but not limited to phasecontrast velocity-encoded [3], tagging [4,5], displacement encoding with stimulated echoes [6], and strain encoding [7] imaging are developed to accurately measure one-dimensional (1D) or two-dimensional (2D) strain. These methods have been validated in phantoms, animals, healthy subjects, and patients, and have demonstrated high accuracy and reproducibility in assessment of regional strain [8]. Since 1D and 2D assessment of regional strain requires simplified geometric assumptions on cardiac geometry and deformation, three-dimensional (3D) approaches have more recently been developed and reported to be in agreement with conventional 2D techniques. However, the first $3 \mathrm{D}$ approaches required prolonged examinations or sophisticated analysis methods often with extensive manual interactions [8]. Although recent improvements in these techniques, e.g. [9-12], provide better solutions, common practice is still focused on 1D strain (predominantly circumferential strain) [13-16] or 2D strain (predominantly circumferential and radial strains) [17-19], in part due to undocumented benefit of fully 3D strain analysis.

A primary purpose of the present study was to test the hypothesis that quantification of 3D strain is superior to that of 1D (circumferential) and 2D (circumferential and radial) strain quantifications in depiction of regional mechanics and discrimination of viable and nonviable myocardial regions. Accordingly, we studied the contractile mechanics of an in vivo porcine model of myocardial infarction (MI). We employed z-encoding harmonic phase (zHARP) [20] and late gadolinium enhancement (LGE) imaging to measure regional strain and myocardial viability, respectively. Univariate, bivariate, and multivariate analyses of regional strain were performed to investigate the diagnostic accuracy of $3 \mathrm{D}$ strain analysis compared with 1D and 2D analyses.

\section{Methods}

Animal studies complied with the Institutional Animal Care and Use Committee and conformed to Guide for the Care and Use of Laboratory Animals.

\section{Experimental preparation}

MI was induced in 20 young farm pigs weighing 25 to $35 \mathrm{~kg}$ as previously described [21]. In brief, an angioplasty balloon was inserted into the left anterior descending coronary artery (LAD) using an over-the-wire technique under fluoroscopic guidance and inflated to a location just distal to the second diagonal branch of the
LAD. Occlusion of the artery was terminated after 150 minutes by deflating the balloon. Post-procedure electrocardiographs showed no prolongation of QRS duration or bundle branch block. All animals were returned to vivarium and received post-operative pain control. Surviving animals, all progressed to heart failure, were monitored daily for the period of the study.

\section{Image acquisition}

CMR images were acquired at $11 \pm 6$ days (baseline) preMI, and at $11 \pm 4$ days (early) and $34 \pm 8$ days (late) postMI. Animals were pre-medicated intramuscularly with droperidol and fentanyl citrate (Innovar $0.1 \mathrm{ml} / \mathrm{kg}$ ), anesthetized with sodium pentobarbital $(20-25 \mathrm{mg} / \mathrm{kg}$, IV), and mechanically ventilated with $1.5 \%$ to $2 \%$ inhalational Isoflurane for the duration of imaging. Images were obtained using a commercial 3.0 T MR scanner (Achieva, Philips Healthcare, Best, NL) equipped with a six-channel cardiac coil. Animals were placed head first and supine with electrodes for vector electrocardiogram gating [22] on the chest used for respiratory gating. Three types of images were acquired in all animals.

\section{Cine}

Using multi-slice steady-state free-precession sequence, cine images were obtained during breathholds to study global left ventricular (LV) function. A total of 9 to 12 contiguous short-axis slices were prescribed to cover the LV from base to apex. Image parameters were the following: repetition time $(\mathrm{TR})=3.4 \mathrm{~ms}$, echo time $(\mathrm{TE})=1.69 \mathrm{~ms}$, flip angle $(\mathrm{FA})=40^{\circ}$, field-ofview $(\mathrm{FOV})=280 \times 280 \mathrm{~mm}^{2}$, matrix size $=256 \times 256$, slice thickness $=8 \mathrm{~mm}$ (with no gap between slices), and temporal resolution $=20 \mathrm{~ms}$. A total of 39 phases were acquired to cover one cardiac cycle.

\section{LGE}

Contrast enhanced images were obtained 10 to 15 minutes after intravenous injection of a bolus of $0.2 \mathrm{mmol} / \mathrm{kg}$ body weight Gadodiamide (Omniscan GE Healthcare Technologies, Chalfont St. Giles, UK) using a Look-Locker inversion recovery sequence [23] and a 3D free-breathing navigator-gated phase-sensitive inversion recovery (PSIR) gradient echo sequence [24]. Images were acquired at 30 contiguous slices with the same orientation used for the cine images. The PSIR image parameters were: TR = $4.6 \mathrm{~ms}, \mathrm{TE}=2.2 \mathrm{~ms}, \quad \mathrm{FA}=15^{\circ}, \quad \mathrm{FOV}=300 \times 300 \mathrm{~mm}^{2}$, matrix size $=256 \times 256$, and slice thickness $=3 \mathrm{~mm}$ (with no gap between slices). The optimal inversion recovery time for optimal suppression of the healthy myocardium was determined by the visual inspection of the LookLocker images, and it ranged between $200 \mathrm{~ms}$ to $250 \mathrm{~ms}$. 


\section{CMR tagging}

zHARP images [20] were acquired during breathholds on the same 9 to 12 short-axis slices used for cine images. This imaging technique, a recently developed and validated tagging technique and an extension to complementary spatial modulation of magnetization [25] and harmonic phase (HARP) analysis [26], enables 3D tracking of shortaxis slices using simultaneous in-plane and through-plane displacement encodings and yields 3D strain measures at every pixel in the imaged slices. The images were acquired using a vector electrocardiogram triggered spoiled gradient echo sequence with segmented $k$-space spiral acquisition and spectral-spatial excitation. Image parameters were: $\mathrm{TR}=20 \mathrm{~ms}, \mathrm{TE}=2.5 \mathrm{~ms}, \mathrm{FA}=15^{\circ}$, FOV $=320 \times 320$ $\mathrm{mm}^{2}$, matrix size $=256 \times 256$, slice thickness $=8 \mathrm{~mm}$ (with no gap between slices), spiral interleaves $=12$, acquisition window $=12 \mathrm{~ms}, \mathrm{z}$-encode frequency $=2 \pi / 33 \mathrm{~mm}$ [27], and tag spacing $=7 \mathrm{~mm}$. On average, a total of 40 phases were acquired to cover one cardiac cycle. Horizontally and vertically tagged images were acquired in two breathholds per slice.

\section{Data analysis}

Cine and LGE images were analyzed using QMass MR software (Medis, Leiden, NL). LV epicardial and endocardial borders, excluding papillary muscles, were traced on end-diastolic and end-systolic cine short-axis images to measure global function. The semi-automated full-widthat-half-maximum method was employed to identify the infarct zone on LGE images [28]. The threshold was adjusted, using n-standard deviations $(\mathrm{n}<4)$ above remote region [28], in a few images where the semi-automated method failed to accurately identify the viable or nonviable regions. Throughout the circumference of the myocardium, infract regions occupying more than $50 \%$ of the chord connecting the endocardial contour to the epicardial contour were marked as transmural [29]. LV volume, mid-ventricular wall thickness, stroke volume, ejection fraction, and scar percentage were computed. The cine and LGE images were analyzed prior to the strain analysis, in a blinded manner. For each zHARP slice, the closest LGE slice was identified and mapped to its corresponding mid-diastolic zHARP image using an affine registration [30]. Epicardial and endocardial borders, the anteroseptal right ventricular attachment point (ARVP) to the LV, and the transmural infarct border points previously marked on LGE images were transferred and matched to the mid-diastolic zHARP image. Using HARP tracking [26], those landmarks were identified for all the zHARP cardiac phases. Figure 1 illustrates epicardial and endocardial borders and the transmural infarct border points on an example LGE and five zHARP images at selected cardiac phases.

\section{Categorization of myocardial segments}

Short-axis slices were categorized into apical, mid, and basal thirds perpendicular to the LV long axis [31] and divided into segments based on viability information provided by the LGE images as shown in Figure 1. The myocardial tissue enclosed circumferentially between the borders of transmural infarct was called the infarct segment. The remaining myocardium was divided into segments with equal circumferential width (five segments in basal and mid slices, and three segments in apical slices). The two segments closest to the infarct were called adjacent segments and the remaining segments were called remote segments. Segments adjacent to an infarct segment in a lower or upper slice were also considered adjacent segments. Baseline (pre-MI) images and slices with no evidence of enhancement were divided equally using the ARVP point similar to the 16-segment model [31] (six segments in basal and mid slices, and four segments in apical slices). Segments at baseline were called healthy segments.

\section{Regional strain endpoints}

zHARP images were analyzed with a software developed in-house using MATLAB (MathWorks, Natick, MA). 3D
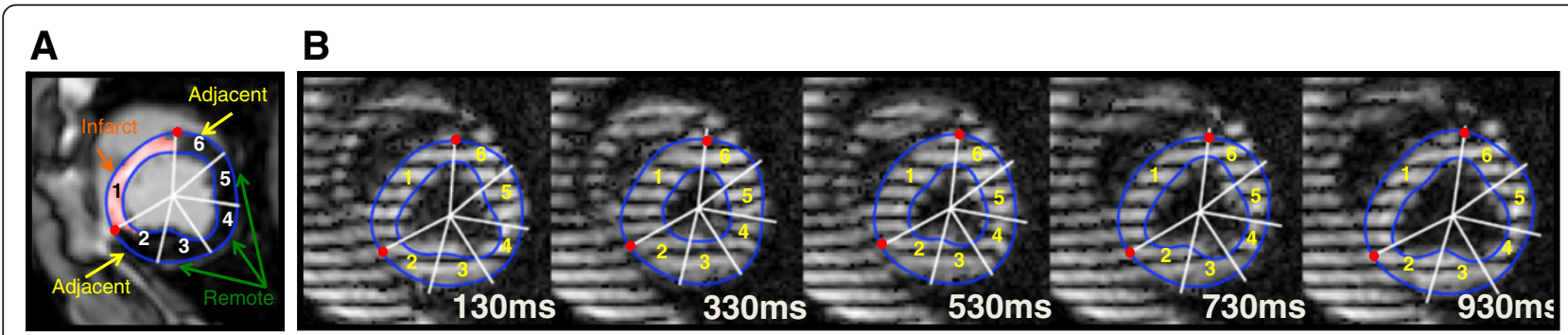

Figure 1 Division of myocardium into circumferential segments. (A) Epicardial and endocardial borders (blue contours), and borders of transmural MI (red points) are delineated on the LGE image. Myocardial tissue enclosed between MI points is called the infarct segment. The rest of the myocardium is divided into equal segments. Infarct: segment 1; Adjacent: segment 2, and 6; Remote: segment 3, 4, and 5. (B) Landmarks delineated on the LGE image are mapped and located on all zHARP cardiac phases by HARP tracking and myocardium is divided into segments accordingly. Five cardiac phases are selected for demonstration. 
displacement and strain tensors were computed for each pixel of the imaged slices during one cardiac cycle as previously reported [27]. Subsequently, the 3D strain tensors were decomposed into three directional strains (circumferential: $E_{c c}$, longitudinal: $E_{l l}$, radial: $E_{r r}$ ) based on the gross anatomy of the heart and the orientation of overlying epicardial surface [32]. In addition, 3D strain tensors were decomposed into three principal strains $\left(E_{1}, E_{2}, E_{3} ; E_{1} \geq E_{2} \geq E_{3}\right.$ ) corresponding to the principal directions of deformation. $E_{1}$ was positive and corresponded to lengthening while $E_{3}$ was negative and corresponded to shortening. $E_{2}$, depending on its sign, corresponded to lengthening or shortening. Note that the directional and principal strains are two representations of the 3D strain tensor. Finally, the abovementioned strain indices were averaged in each segment.

\section{Statistical analysis}

Data was reported as mean \pm one standard deviation (SD). One-way analysis of variance (ANOVA) was used

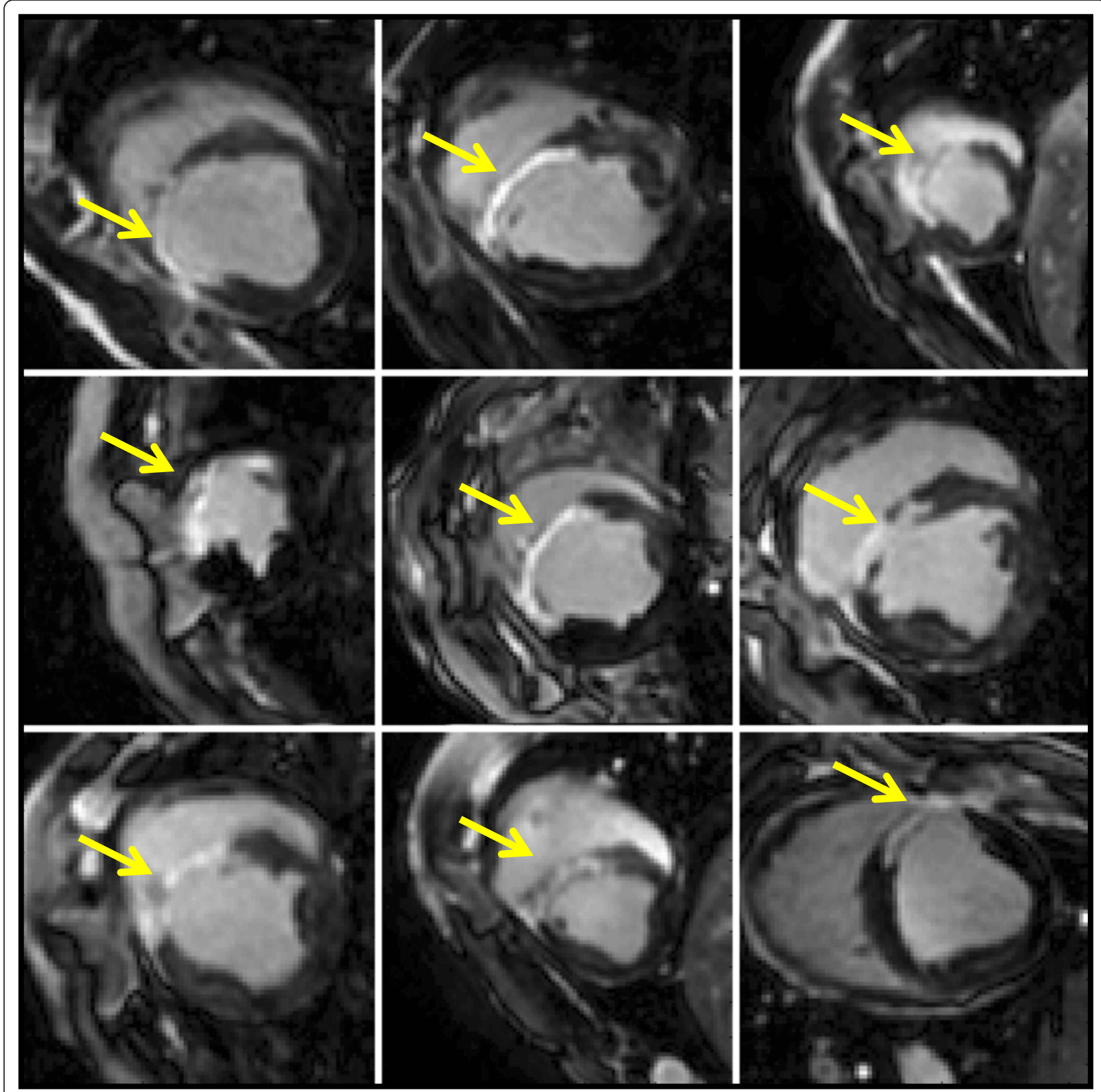

Figure 2 Example LGE images in nine animals. Myocardial infarction is mainly located in the anterior wall and anterior septum with transmurality greater than $90 \%$ of the wall thickness (yellow arrows). Isolated infarct zones, and slices with sub-endocardial infarct and no presence of transmural infarct were not observed in the entire dataset. 
Table 1 Global characteristics of left ventricles

\begin{tabular}{lccc}
\hline & Baseline & Early & Late \\
\hline Days after MI & - & $11 \pm 4$ & $34 \pm 8$ \\
LVEDV (ml) & $52.5 \pm 8.9$ & $72.9 \pm 14.2$ & $91.2 \pm 27.9^{*}+$ \\
LVESV $(\mathrm{ml})$ & $29.7 \pm 5.9$ & $51.0 \pm 13.1$ & $58.6 \pm 20.6^{*}$ \\
LVSV $(\mathrm{ml})$ & $22.8 \pm 3.3$ & $21.8 \pm 9.4$ & $32.6 \pm 8.7^{*}+$ \\
LVEF $(\%)$ & $43.6 \pm 2.8$ & $26.7 \pm 7.6^{*}$ & $37.5 \pm 3.9+$ \\
Scar $(\%)$ & - & $16.2 \pm 4.9$ & $16.5 \pm 4.0$
\end{tabular}

Values are mean $\pm S D . L V E D V=$ left ventricular end-diastolic volume; $L V E S V=$ left ventricular end-systolic volume; $L V S V=$ left ventricular stroke volume; $L V E F=$ left ventricular ejection fraction. ${ }^{*} p<0.05$ vs. baseline; $t p<0.05$ vs. early.

to compare LV volume, stroke volume, and ejection fraction at baseline and post-MI time points. Early and late post-MI scar percentages were compared using a paired Student $t$-test. Two-way repeated measures ANOVA was used to compare wall thickness at two cardiac phases (end-systole and end-diastole) and at baseline and postMI time points. Similarly, two-way repeated measures ANOVA was used to compare strain at the three myocardial segments (infarct, adjacent, and remote) and the two post-MI time points. In addition, ANOVA was used to compare the post-MI strain measures with their baseline values. A Tukey test was used for post hoc analysis if ANOVA was positive.

Post-MI time points were grouped together and univariate $\left(E_{c c}\right)$, bivariate $\left(E_{c c}\right.$ and $\left.E_{r r}\right)$, and multivariate $\left(E_{c c}\right.$, $E_{r n}$ and $\left.E_{l l}\right)$ stepwise logistic regression analyses were performed to evaluate the relation between the strain variables in the healthy segments and the infarct segments, as well as in the adjacent segments. Variables identified with a p-value $<0.05$ based on univariate analysis were entered as covariates in the bivariate and multivariate models. A similar multivariate model was created using the $3 \mathrm{D}$ principal strain variables $\left(E_{1}, E_{2}\right.$,
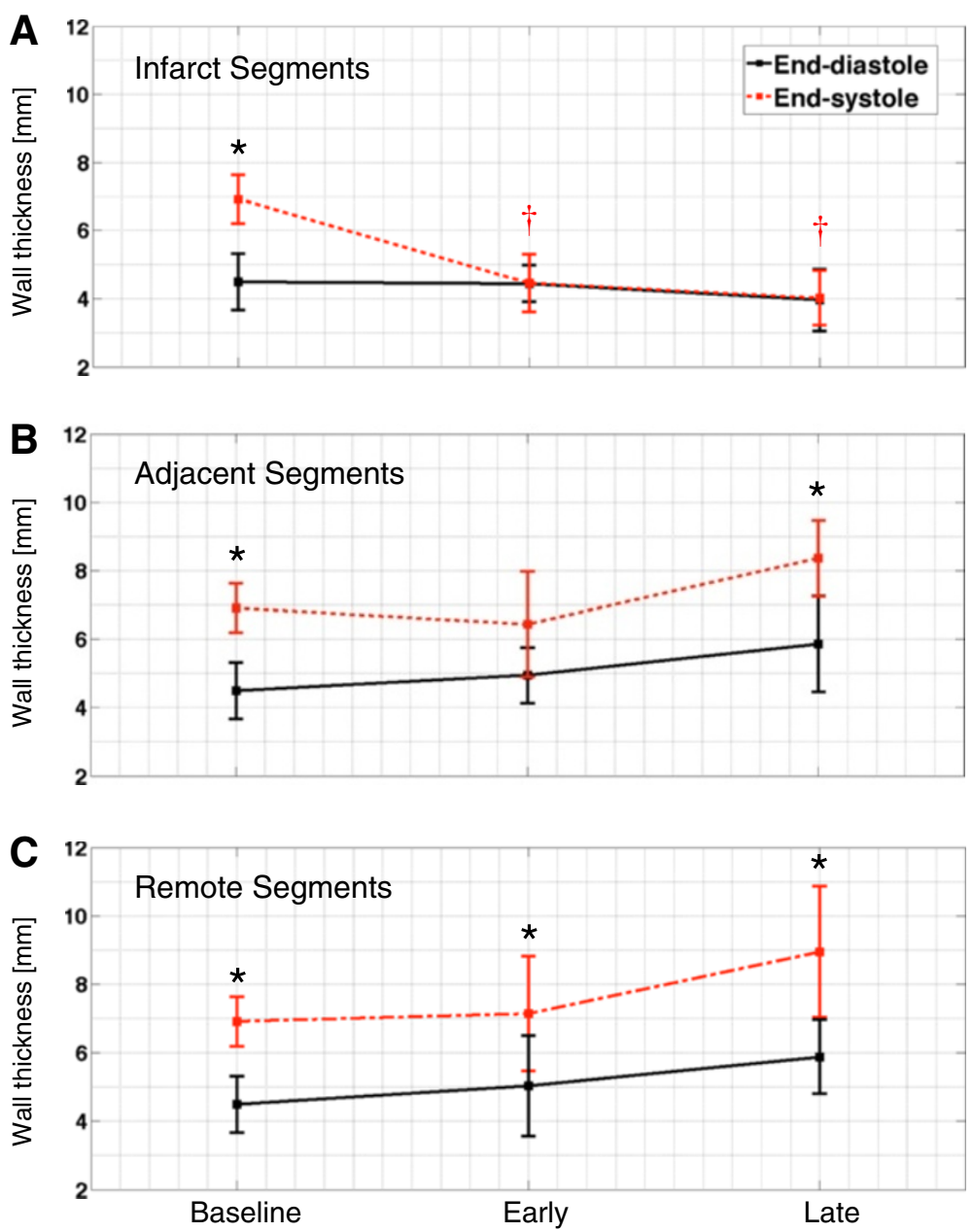

Figure $\mathbf{3}$ LV wall thickness. Mid-ventricular wall thickness is measured in infarct (A), adjacent (B), remote (C) segments at end-systole (red) and end-diastole (black) at baseline and post-MI ( ${ }^{*} p<0.05$ vs. end-diastole, $+p<0.05$ vs. end-systolic baseline.). While no significant wall thickening from end-diastole to end-systole is present in infarct and adjacent segments at early post-Ml, the wall thickening in adjacent segments is improved $(p<0.05)$ at late post-MI. This observation is not present in Ml segments. 
and $\left.E_{3}\right)$. A receiver operating characteristic (ROC) analysis was used to summarize the overall diagnostic accuracy of the strain models in detecting infarct and adjacent segments from healthy segments. The incremental value of multivariate models was compared with univariate and bivariate models by pair-wise comparison of the c-statistics, equal to the area under the ROC curves using Delong's method [33]. In all tests, a p-value $<0.05$ was considered statistically significant. Statistical analyses were performed using JMP (SAS Institute Inc., Carey, NC) and MedCalc (Mariakerke, Belgium).

\section{Results}

Of 20 animals, 5 died due to incessant ventricular fibrillation (one died intra-operatively during balloon occlusion and four died during the immediate post-operative period). Surviving animals all completed the imaging protocol. Average heart rate was 79 beats/min. Average breathhold durations were $17 \mathrm{~s}$ and $18 \mathrm{~s}$ for cine and zHARP imaging, respectively.

\section{Characterization of the infarct model}

LGE images revealed transmural infarcts in the apical to mid slices and mainly in the anterior wall and anterior septum. Transmurality was greater than $90 \%$ of wall thickness and the extension of sub-endocardial infarct was smaller than $25 \%$ of the area of adjacent segments in all animals. Isolated infarct zones, and slices with subendocardial infarct and no presence of transmural infarct were not observed in the entire dataset. A subset of LGE images is illustrated in Figure 2. Scar size was approximately $16 \%$ of LV volume and did not display variation post-MI (early: $16.2 \pm 4.9 \%$ vs. late: $16.5 \pm 4.0 \%, \mathrm{p}=0.96)$.

\section{LV global function and wall thickness}

Table 1 summarizes LV global function at baseline and post-MI time points. Increases in stroke volume and LV volume at both end-systole and end-diastole were observed post-MI. Ejection fraction reduced from baseline to early post-MI $(43.6 \pm 2.8 \%$ vs. $26.7 \pm 7.6 \%, \mathrm{p}=$ $0.0105)$ and then increased at late post-MI $(26.7 \pm 7.6 \%$ vs. $37.5 \pm 3.9 \%, \mathrm{p}=0.0385$ ).

Mid-ventricular wall thickness values at end-systole and end-diastole are shown in Figure 3. In remote segments, wall thickness at end-systole increased significantly from that at end-diastole at all times $(\mathrm{p}<0.001)$. In addition, a progressive increasing trend in the wall
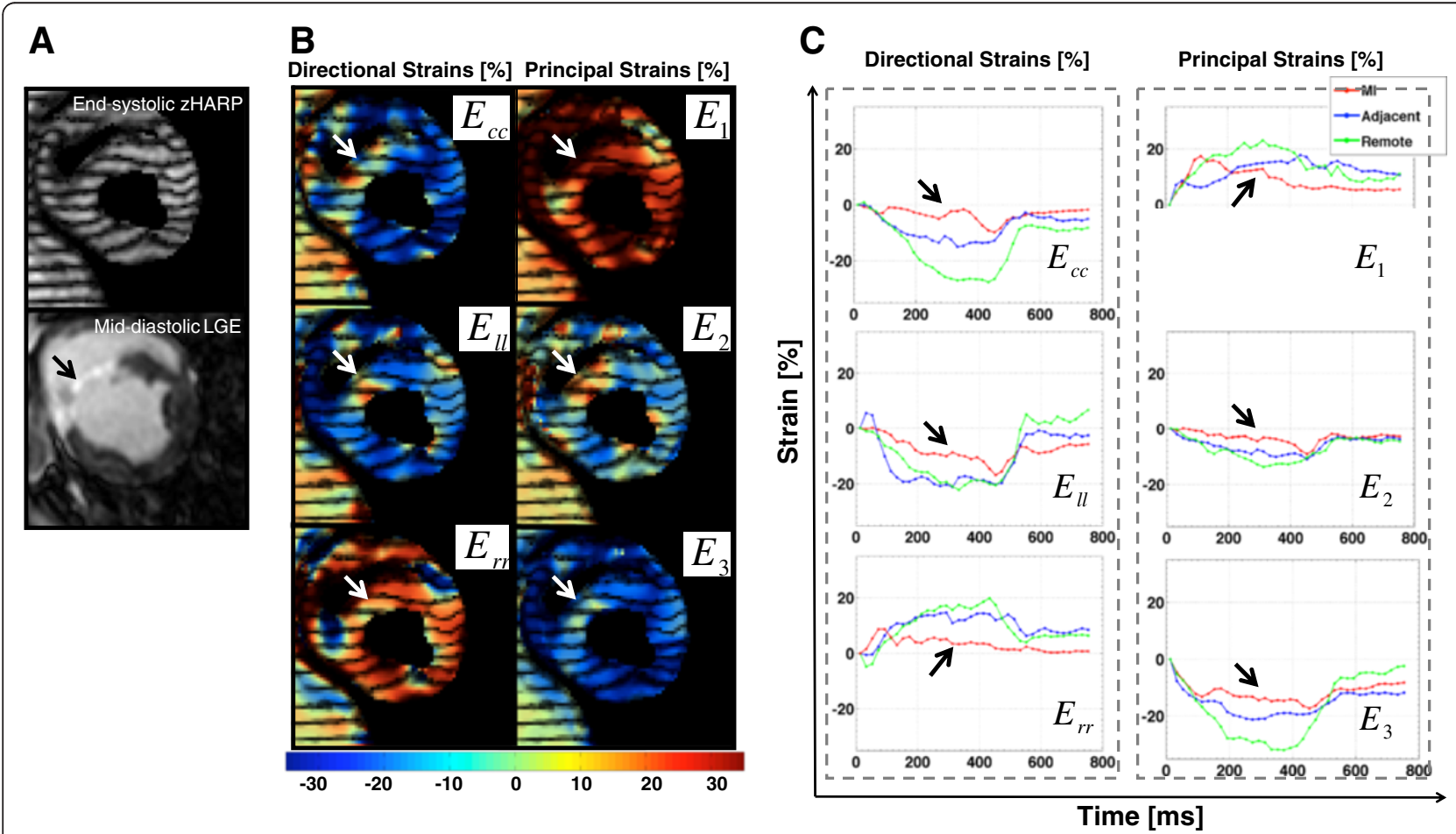

Figure 4 Example mid-ventricular slice at early post-MI. (A) End-systolic zHARP tagging image (top) with paired mid-diastolic LGE image (bottom). (B) End-systolic directional and principal strains overlaid on the zHARP image. Variation of the strain in infarct region from the remote region is consistent with enhancement in LGE image (white arrows). (C) Average directional strains $\left(E_{c c}=\right.$ circumferential strain, $E_{\| l}=$ longitudinal strain, $E_{r r}=$ radial strain), and principal strains $\left(E_{1}, E_{2}, E_{3}\right)$ are displayed in the infarct, adjacent, and remote segments in one cardiac cycle. 
thickness of remote segments was observed from baseline to late post-MI $(\mathrm{p}=\mathrm{NS})$ at both end-systole and end-diastole (Figure 3C). In contrast, a significant increase of end-systolic wall thickness from that of enddiastolic, in adjacent segments, was only observed at baseline and late post-MI $(\mathrm{p}<0.05)$. In MI segments, however, there was no post-MI wall thickening from end-systole to end-diastole $(\mathrm{p}=\mathrm{NS})$; moreover, endsystolic wall thickness in these segments decreased significantly from baseline values $(\mathrm{p}<0.0001)$.

\section{D Regional strain}

Figure 4A shows an example of an end-systolic zHARP image and its corresponding mid-diastolic LGE image in one animal at early post-MI. Directional and principal strains overlaid on the zHARP image and their temporal profile are shown in Figure $4 \mathrm{~B}$ and $4 \mathrm{C}$, respectively. Reduced magnitudes were observed in all strain indices in infarct segments versus adjacent and remote segments (Figure 4C, black arrows).
Myocardial 3D regional strain values at baseline and post-MI time points are listed in Table 2 . Regional strain values did not demonstrate significant changes in remote segments at both post-MI time points as compared to the baseline healthy segments $(\mathrm{p}=\mathrm{NS})$. Directional and principal 3D strain magnitudes reduced significantly in adjacent segments compared to their baseline values as well as that of the remote segments $(\mathrm{p}<0.05)$ at both post-MI time points. A similar finding was observed in infarct segments $(\mathrm{p}<0.05)$. In addition, $E_{c c}, E_{r n}, E_{2}$, and $E_{3}$ reduced significantly in infarct segments compared with that in the adjacent segments $(\mathrm{p}<0.05)$. The twoway ANOVA analysis revealed no significant changes between the early and late post-MI time points in any of the six strain measures.

Identification of infarct segments by univariate, bivariate, and multivariate models

All the three directional strains proved significant factors $(\mathrm{p}<0.05)$ in distinguishing infarct segments from

Table 2 3D Regional strain [\%] at baseline and post-MI time points

\begin{tabular}{|c|c|c|c|c|c|}
\hline & & Healthy & Remote & Adjacent & Infarct \\
\hline \multirow[t]{12}{*}{ Directional strains } & $E_{c c}$ & & & & \\
\hline & Baseline & $-19.6 \pm 3.7$ & & & \\
\hline & Early & & $-21.0 \pm 5.5$ & $-10.8 \pm 8.8^{*}+$ & $-3.9 \pm 3.7^{*} \dagger \neq$ \\
\hline & Late & & $-20.1 \pm 5.1$ & $-11.8 \pm 4.7^{*} \dagger$ & $-2.5 \pm 4.1^{*} \dagger \neq$ \\
\hline & $E_{r r}$ & & & & \\
\hline & Baseline & $16.4 \pm 3.3$ & & & \\
\hline & Early & & $16.1 \pm 4.7$ & $8.8 \pm 5.2^{*} \dagger$ & $5.1 \pm 5.1^{*} \dagger$ \\
\hline & Late & & $16.8 \pm 3.9$ & $7.3 \pm 4.8^{*}+$ & $1.6 \pm 3.2^{*}+\neq$ \\
\hline & $E_{I I}$ & & & & \\
\hline & Baseline & $-21.7 \pm 6.7$ & & & \\
\hline & Early & & $-20.3 \pm 5.8$ & $-10.9 \pm 6.1^{*} \dagger$ & $-8.2 \pm 4.2^{*}+$ \\
\hline & Late & & $-19.1 \pm 7.4$ & $-10.0 \pm 5.1^{*}+$ & $-4.3 \pm 5.8^{*}+$ \\
\hline \multirow[t]{12}{*}{ Principal strains } & $E_{1}$ & & & & \\
\hline & Baseline & $24.3 \pm 7.7$ & & & \\
\hline & Early & & $28.1 \pm 6.8$ & $19.2 \pm 6.1^{*}+$ & $15.5 \pm 2.0^{*}+$ \\
\hline & Late & & $26.8 \pm 7.0$ & $17.3 \pm 5.6^{*}+$ & $12.8 \pm 4.3^{*}+$ \\
\hline & $E_{2}$ & & & & \\
\hline & Baseline & $-13.1 \pm 3.1$ & & & \\
\hline & Early & & $-12.9 \pm 3.5$ & $-7.3 \pm 3.9^{*}+$ & $-3.3 \pm 2.7^{*}+\neq$ \\
\hline & Late & & $-12.0 \pm 4.3$ & $-6.8 \pm 3.3^{*}+$ & $-3.4 \pm 2.8^{*} \dagger \neq$ \\
\hline & $E_{3}$ & & & & \\
\hline & Baseline & $-25.7 \pm 4.6$ & & & \\
\hline & Early & & $-27.8 \pm 4.2$ & $-20.3 \pm 4.4^{*} \dagger$ & $-12.8 \pm 2.4^{*} \dagger \neq$ \\
\hline & Late & & $-26.0 \pm 6.1$ & $-20.3 \pm 3.3^{*} \dagger$ & $-13.9 \pm 2.7^{*} \dagger \neq$ \\
\hline
\end{tabular}

Values are mean $\pm S D$. ${ }^{*} p<0.05$ vs. healthy; $\uparrow p<0.05$ vs. remote; $\neq p<0.05$ vs. adjacent. 
healthy segments by univariate analysis and thus they were entered into the bivariate and multivariate models. The 3D (using $E_{c c}, E_{r n}$ and $E_{l l}$ ) and 2D (using only $E_{c c}$ and $E_{r r}$ ) models demonstrated modest incremental improvement in c-statistic compared with the $E_{c c}$ univariate model (3D: 0.996, SE, 0.004, CI, 0.9641.000; 2D, 0.991, SE, 0.006, CI, 0.956-1.000; 1D, 0.986, SE, 0.010, CI, 0.949-0.999, p = NS, Figure 5A). As shown by ROC curves in Figure $6 \mathrm{~A}, E_{c c}$ alone provided a powerful metric with a large diagnostic accuracy (95\%) in distinguishing infarct segments. The 2D and 3D strain models demonstrated $94 \%$ and $98 \%$ accuracy, respectively. Similarly, the multivariate model using 3D principal strains, the alternate representation of 3D strain tensor, demonstrated a large c-statistic (0.997, SE, 0.003, CI, 0.964-1.000) in detection of infarct segments with no significant difference from the multivariate model using directional strains $(\mathrm{p}=\mathrm{NS})$.

\section{Identification of adjacent segments by univariate,} bivariate and multivariate models

Univariate analysis demonstrated that the three directional strains are independently significant $(\mathrm{p}<0.001)$ in detection of adjacent segments. The incremental value of $2 \mathrm{D}$ and $3 \mathrm{D}$ models in comparison to $1 \mathrm{D} E_{c c}$ model is shown in Figure 5B. Diagnostic accuracies of all strain models in detecting adjacent segments are smaller than those associated with detection of infarct segments. However, the bivariate model using $E_{c c}$ and $E_{r r}$ in which both appeared as significant factors $(\mathrm{p}<$ $0.001)$, demonstrated improved c-statistic $(\mathrm{p}=0.0009)$ compared with 1D univariate analysis using $E_{c c}$ only (2D: c-statistic, 0.941, SE, 0.020, CI, 0.887-0.974; 1D: c-statistic, 0.846, SE, 0.035, CI, 0.774-0.902). Furthermore, all the strain variables $\left(E_{c c}, E_{r n}\right.$ and $\left.E_{l l}\right)$ were independently significant $(\mathrm{p}<0.001)$ in the $3 \mathrm{D}$ analysis and this multivariate model demonstrated even higher diagnostic accuracy (c-statistic, 0.981, SE, 0.010, CI, 0.941-0.997, $\mathrm{p}=0.0055$ ). ROC curves for these strain models are shown in Figure 6B. The 1D $E_{c c}$ model resulted in modest $71 \%$ diagnostic accuracy, which was improved to $81 \%$ and $96 \%$ by $2 \mathrm{D}$ and 3D models, respectively. Lastly, the 3D analysis using principal strain variables demonstrated a comparable c-statistic to directional strains in detection of adjacent segments (cstatistic, 0.971, SE, 0.012, CI, 0.926-0.992, $\mathrm{p}=\mathrm{NS}$ ).
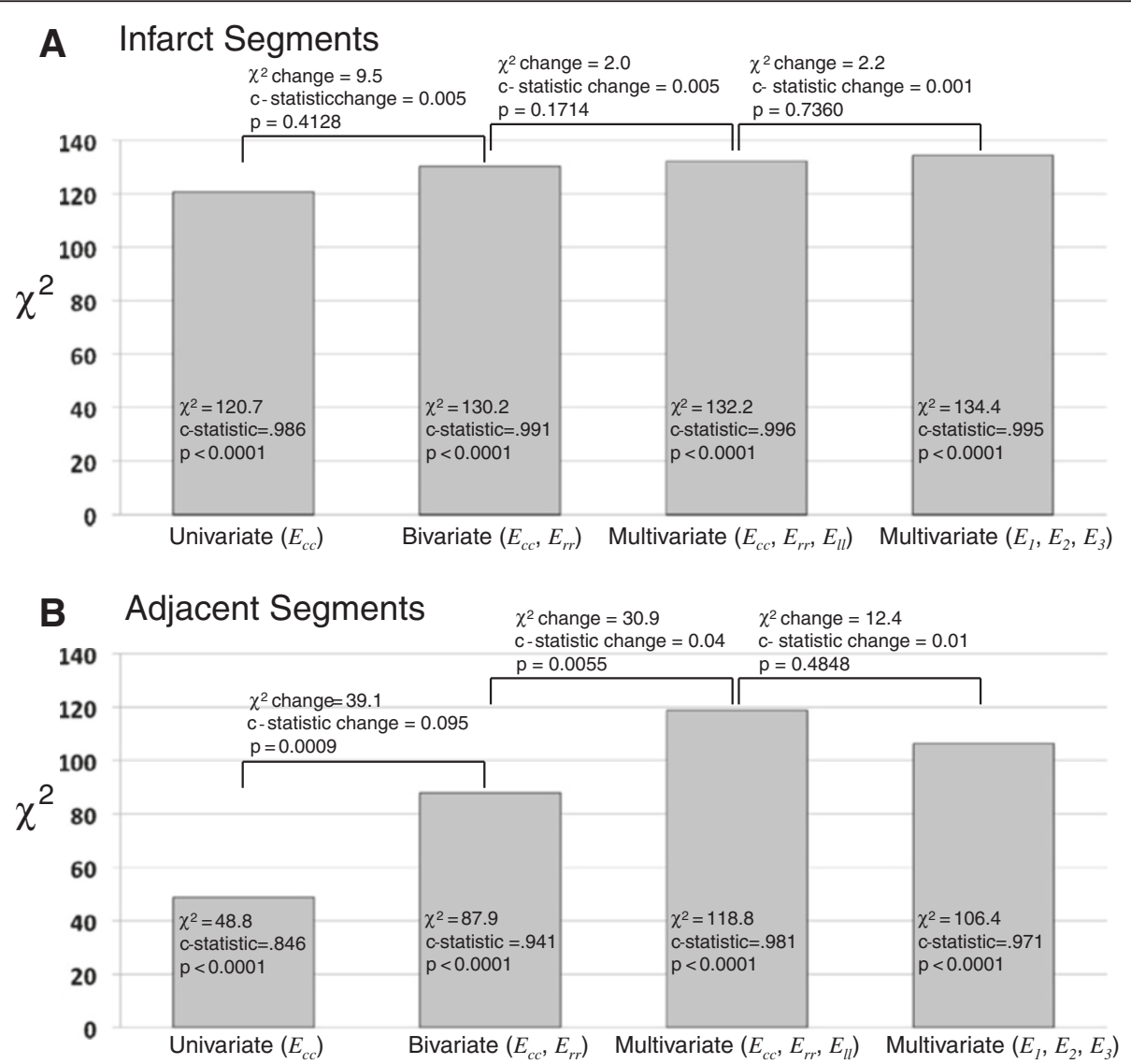

Figure 5 Incremental value of myocardial strain multivariate 3D models over 2D and 1D models in identifying infarct (A) and adjacent (B) segments from healthy segments. 

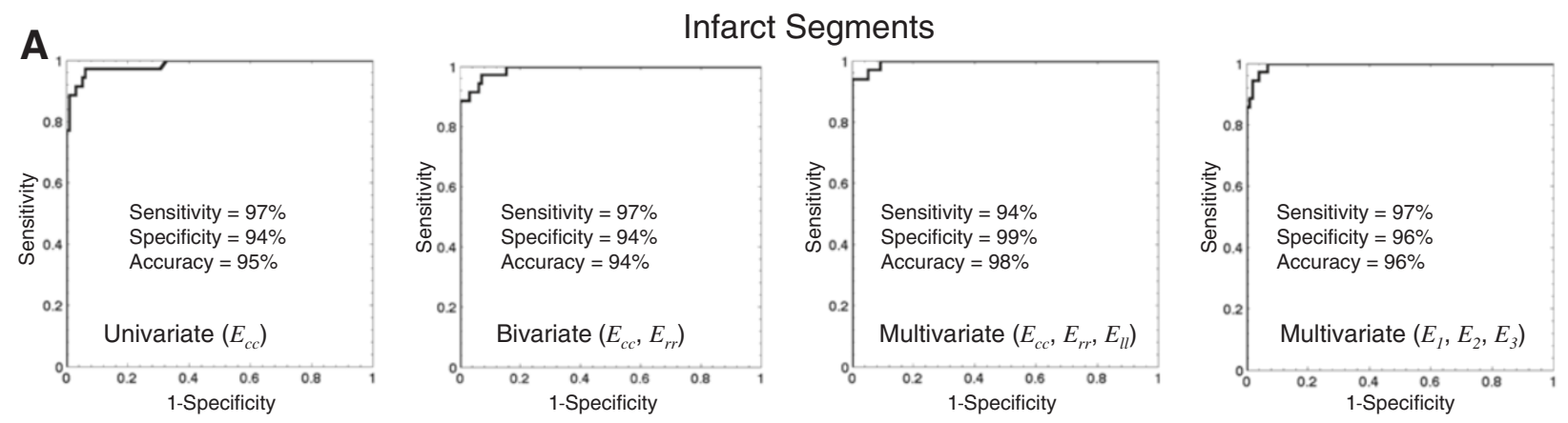

B
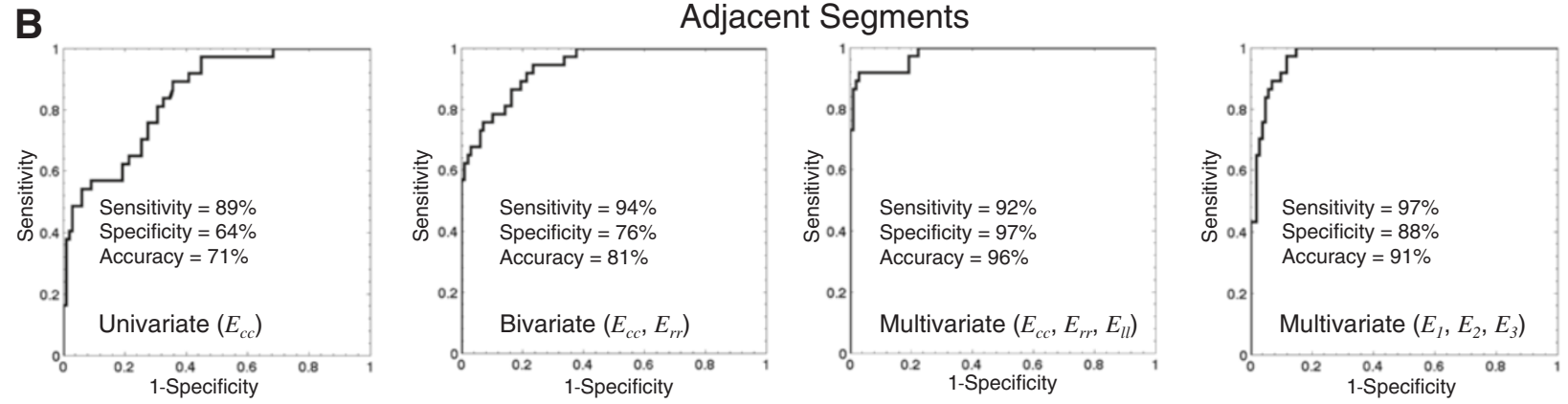

Figure 6 Receiver operating characteristic curves, testing the accuracy of regional strain models in identifying infarct (A) and adjacent (B) segments from healthy segments.

\section{Discussion}

Advances in CMR [9-12] allow comprehensive 3D quantification of myocardial contractility without the simplified geometric assumptions commonly associated with 1D or 2D techniques. The clinical adoption of 3D methods however has been hindered in part due to increased imaging time and processing steps required by majority of such methods. Recent developments in CMR imaging and analysis aim to address these issues, and decrease the scanning and processing durations. zHARP, in particular, enables 3D tracking of short-axis slices using simultaneous in-plane and through-plane displacement encodings without affecting the duration of image acquisition. As a result, imaging multiple orientations is eliminated and zHARP yields $3 \mathrm{D}$ strain measures at every pixel in the imaged slices without the need for image registration or numerical interpolations [27]. Using zHARP, the present study was designed to assess the value of $3 \mathrm{D}$ regional mechanics analysis over the usual in-plane analysis. This incremental value can potentially facilitate the clinical adoption of $3 \mathrm{D}$ methods.

In this study, we used a porcine model of MI [34] in which transmural infarction was present in the apical to mid levels. Mean infarct volume was $16 \%$ of LV volume with no post-MI variations at 11 days and one month $(\mathrm{p}=$ NS). Additionally, classic features of post-MI remodeling was demonstrated in the wall and ventricular chambers (Table 1), accompanied with wall thinning in infarct segments, and wall thickening in remote segments (Figure 3). In the adjacent segments, which contained no transmural infarct and very little sub-endocardial infarct, the wall thickness measurements showed no thickening at early post-MI. Wall thickening in these segments however improved at the late time point. Unlike previous research that reports a decrease in ejection fraction after LV remodeling, an increase in ejection fraction was observed at late post-MI. This observation can be due to mitral valve regurgitation.

For regional strain assessment, the myocardium was divided into segments based on the transmurality and distribution of infarct zone over the circumference of myocardium, characterized by LGE images (Figure 1). Unlike the standard 16-segment model, this method of segmentation eliminated segments with partial transmural infarcts and enabled us to independently study strain patterns in transmural infarcts and the peri-infarct regions that demonstrate different post-MI mechanical behaviors [35]. The transmural infarct consistently spanned the anterior and anterior septal segments, as the infarct was generated following the same procedure and occlusion location in all animals. The size of infarction thereby was largely consistent with a relatively small variation among segment sizes at each cardiac level.

While 3D strain measures in remote segments did not vary significantly from that of the pre-MI healthy segments, the transmural infarct segments and their 
adjacent segments demonstrated significantly smaller values of 3D strain compared to the remote and healthy regions. Subsequently, univariate, bivariate, and multivariate regression analyses demonstrated that all the directional strain indices $\left(E_{c c}, E_{r n}\right.$ and $\left.E_{l l}\right)$ are significant covariates in identification of adjacent and infarct segments from healthy counterparts. The multivariate model using $E_{c c}, E_{r n}$ and $E_{l l}$ resulted in significant improvement $(\mathrm{p}<0.01)$ in $\mathrm{c}$-statistic and diagnostic accuracy (c-statistic $=0.981$, accuracy $=96 \%$ ) compared with the 2D model using $E_{c c}$ and $E_{r r}$ (c-statistic $=0.941$, accuracy $=81 \%$ ), and the univariate model using $E_{c c}$ (cstatistic $=0.846$, accuracy $=71 \%$ ). This finding shows that 3D strain may therefore allow a more accurate and reliable mapping of regional contractility in infarct neighboring regions in comparison with commonly employed 1D or 2D strain measures. However, the 3D strain analysis (cstatistic $=0.996$, accuracy $=98 \%$ ) did not appear significantly superior to simplified single-plane quantification of strain (2D: c-statistic $=0.991$, accuracy $=94 \%, 1 D$ : cstatistic $=0.986$, accuracy $=95 \%)$ in the presence of a transmural infarct.

The 3D principal strain indices $\left(E_{1}, E_{2}\right.$, and $\left.E_{3}\right)$ corresponded to the three directions of maximal myocardial deformation. $E_{1}$, corresponding to maximum thickening, was always larger than radial thickening not only in the infarct segments but also in the remote, and adjacent segments (Table 2). Similarly, the maximum shortening values, $E_{3}$, were larger (in absolute value) than the corresponding circumferential strain values in all segments at all time points. These principal strain indices demonstrated a precise assessment of regional function and did not illustrate inferior diagnostic performance in comparison with the more commonly used directional strain indices (Figure 6). The decomposition of principal strain indices from 3D strain tensor, unlike directional strain indices, does not depend on the gross geometry of LV and the orientation of imaged slice. This universal aspect of principal strains and their insensitivity to image planning may result in more accurate and robust strain values in comparison to $E_{c c}, E_{r n}$ and $E_{l l}$. However, this speculation requires a more careful study to be confirmed. The direction of principal strain may also provide a regionally varying index of myocardial deformation. However, the extraction of angles and averaging those angles within each segment must be performed under special care especially in regions with infarct, which may lack a dominant direction of deformation. Definitive conclusion warrants further investigation and analysis, which is beyond the scope of the present work.

CMR-based 3D strain may therefore allow accurate and reliable mapping of regional contractility and assessment of viability. 3D analysis may also have implications beyond analysis of myocardial infarction and it may help in assessing patients with ischemic or non-ischemic cardiomyopathy [36]. The present study may help lay the road for a larger follow up study, with a longer post-MI time period and a wider spectrum of MI size, to investigate if an early strain signature can predict post-MI remodeling and determine the salvageable myocardium.

\section{Limitations}

The MI model used in this study was approximately $16 \%$ of the LV volume and restricted to the apex and septum. Furthermore, the MI was mainly transmural and the extent of sub-endocardial infarct was small $(<25 \%$ area of adjacent segments). Infarctions in clinical practice that are not revascularized in time usually present a wider spectrum of MI size and distribution with the potential for larger sub-endocardial infarcts. The latter condition would present the ideal condition for 3D regional function analysis. The segmentation method for the baseline images and the post-MI images was different, which prevented direct comparison. Also, we did not perform pathologic validation of our model. However, this porcine model of MI has been characterized using high resolution ex vivo CMR [37] and has been reported to exhibit all the morphologic and functional remodeling features of clinical infarctions [34]. There is also extensive published literature on the spatial correlation of infarct and its neighboring regions with delayed enhancement. Similarly, we did not perform a dobutamine challenge to assess the viable segments. This concept also has been validated repeatedly with regards to delayed enhancement. Lastly, we did not assess mitral valve regurgitation, which could potentially explain the increase in ejection fraction at the late post-MI time point.

\section{Conclusions}

Multivariate 3D strain analysis using CMR accurately differentiates infarct from normal myocardium and correlates with delayed enhancement. Furthermore, cumulative $3 \mathrm{D}$ strain is superior to $1 \mathrm{D}$ and $2 \mathrm{D}$ strain analyses for identification of infarct neighboring regions. Further evaluation of this technique in human subjects will help determine the clinical value of $3 \mathrm{D}$ regional strain quantification.

\section{Competing interests}

Jerry L. Prince is a co-founder of Diagnosoft Inc., a company that licensed the HARP technology. The terms of this arrangement are managed by the Johns Hopkins University in accordance with its conflict of interest policies. Other authors have declared no competing interests.

\section{Authors' contributions}

SS: substantial contributions to design of study, analysis and interpretation of data, and drafted the manuscript. KZA: substantial contributions to design of study, acquisition and interpretation of data, and revision of manuscript. TS: substantial contributions to acquisition of data. HA: substantial contributions to interpretation of data. MRA: substantial contributions to design of study, interpretation of data, and revision of manuscript. TPA: substantial contributions to design of study, interpretation of data, and revision of manuscript. JLP: substantial contributions to design of study, interpretation 
of data, and revision of manuscript. All authors read and approved the final manuscript.

\section{Acknowledgements}

We thank Evert-Jan Vonken, MD, PhD, and Michael Schär, PhD for kindly providing the technical expertise with CMR acquisitions, and Amr Youssef, MD for help with animal studies. This work was supported in part by $\mathrm{NIH}$ / NHLBI research grant R01HL47405.

\section{Author details}

${ }^{1}$ Department of Electrical and Computer Engineering, Johns Hopkins University, 3400 N. Charles Street, Clark Hall 201, Baltimore, MD 21218, USA. ${ }^{2}$ National Institute of Diabetes and Digestive and Kidney Diseases, NIH, Bethesda, MD, USA. ${ }^{3}$ Department of Medicine, Division of Cardiology, Johns Hopkins University, Baltimore, MD, USA. ${ }^{4}$ Philips Research North America, Briarcliff Manor, NY, USA. ${ }^{5}$ Department of Radiology and Radiological Science, Johns Hopkins University, 3400 N. Charles Street, Clark Hall 201, Baltimore, MD 21218, USA.

Received: 24 April 2012 Accepted: 28 November 2012 Published: 13 December 2012

\section{References}

1. Antoni ML, Mollema SA, Delgado V, Atary JZ, Borleffs CJW, Boersma E, Holman ER, van der Wall EE, Schalij MJ, Bax JJ: Prognostic importance of strain and strain rate after acute myocardial infarction. Eur Heart J 2010, 31:1640-1647.

2. Ferrari VA, Duffy KJ: Clinical Applications of CMR Techniques for Assessment of Regional Ventricular Function. In: Kwong RY, editor. Cardiovascular Magnetic Resonance Imaging. Humana Press; 2008: p. 155-174.

3. van Dijk P: Direct cardiac NMR imaging of heart wall and blood flow velocity. J Comput Assisted Tomogr 1984, 8:429-436.

4. Axel L, Dougherty L: Heart wall motion: improved method of spatial modulation of magnetization for MR imaging. Radiology 1989, 172:349-350.

5. Zerhouni EA, Parish DM, Rogers WJ, Yang A, Shapiro EP: Human heart: tagging with MR imaging-a method for noninvasive assessment of myocardial motion. Radiology 1988, 169:59-63.

6. Aletras AH, Ding S, Balaban RS, Wen H: DENSE: displacement encoding with stimulated echoes in cardiac functional MRI. J Magn Reson 1999, 137:247-252.

7. Osman NF, Sampath S, Atalar E, Prince JL: Imaging longitudinal cardiac strain on short-axis images using strain-encoded MRI. Magn Reson Med 2001, 46:324-334.

8. Ibrahim E-SH: Myocardial tagging by cardiovascular magnetic resonance: evolution of techniques-pulse sequences, analysis algorithms, and applications. J Cardiovasc Magn Reson 2011, 13:36.

9. Hess AT, Zhong X, Spottiswoode BS, Epstein FH, Meintjes EM: Myocardial $3 \mathrm{D}$ strain calculation by combining cine displacement encoding with stimulated echoes (DENSE) and cine strain encoding (SENC) imaging. Magn Reson Med 2009, 62:77-84.

10. Zhong X, Spottiswoode BS, Meyer CH, Kramer CM, Epstein FH: Imaging three-dimensional myocardial mechanics using navigator-gated volumetric spiral cine DENSE MRI. Magn Reson Med 2010, 64:1089-1097.

11. Li P, Prince JL, Lima JAC, Osman NF: Fast tracking of cardiac motion using 3D-HARP. Biomed Eng, IEEE Trans 2005, 52:1425-1435.

12. Rutz AK, Ryf S, Plein S, Boesiger P, Kozerke S: Accelerated whole-heart 3D CSPAMM for myocardial motion quantification. Magn Reson Med 2008, 59:755-763.

13. Mewton N, Croisille P, Revel D, Weber O, Higgins CB, Saeed M: Left ventricular Postmyocardial infarction remodeling studied by combining MR-tagging with delayed MR contrast enhancement. Invest Radiol 2008, 43:219-228. 210.1097/RLI.1090b1013e318161613e.

14. Hor KN, Wansapura J, Markham LW, Mazur W, Cripe LH, Fleck R, Benson DW, Gottliebson WM: Circumferential strain analysis identifies strata of cardiomyopathy in Duchenne muscular dystrophy: a cardiac magnetic resonance tagging study. J Am Coll Cardiol 2009, 53:1204-1210.

15. Korosoglou G, Lossnitzer D, Schellberg D, Lewien A, Wochele A, Schaeufele T, Neizel M, Steen H, Giannitsis E, Katus HA, Osman NF: Strain-encoded cardiac
MRI as an adjunct for dobutamine stress testing: incremental value to conventional wall motion analysis. Circ Cardiovasc Imaging 2009, 2:132-140.

16. Rosen BD, Edvardsen T, Lai S, Castillo E, Pan L, Jerosch-Herold M, Sinha S, Kronmal R, Arnett D, Crouse JR III, et al.: Left ventricular concentric remodeling is associated with decreased global and regional systolic function: the multi-ethnic study of atherosclerosis. Circulation 2005, 112:984-991.

17. Qiao H, Zhang H, Yamanaka S, Patel W, Petrenko NB, Huang B, Muenz LR, Ferrari VA, Boheler KR, Zhou R: Long-term improvement in Postinfarct left ventricular global and regional contractile function is mediated by embryonic stem cell-derived cardiomyocytes/clinical perspective. Circ Cardiovasc Imaging 2011, 4:33-41.

18. Blom AS, Pilla JJ, Arkles J, Dougherty L, Ryan LP, Gorman lii JH, Acker MA Gorman RC: Ventricular restraint prevents infarct expansion and improves Borderzone function after myocardial infarction: a study using magnetic resonance imaging, three-dimensional surface modeling, and myocardial tagging. Ann Thorac Surg 2007, 84:2004-2010.

19. Dawson DK, Maceira AM, Raj VJ, Graham C, Pennell DJ, Kilner PJ: Regional thicknesses and thickening of compacted and Trabeculated myocardial layers of the normal left ventricle studied by cardiovascular magnetic resonance/clinical perspective. Circ Cardiovasc Imaging 2011, 4:139-146.

20. Abd-Elmoniem KZ, Osman NF, Prince JL, Stuber M: Three-dimensional magnetic resonance myocardial motion tracking from a single image plane. Magn Reson Med 2007, 58:92-102.

21. Abd-Elmoniem $K Z$, Santaularia Tomas M, Sasano T, Soleimanifard S, Vonken E-JP, Youssef A, Agarwal HK, Dimaano V, Calkins H, Stuber M, et al.: Assessment of distribution and evolution of Mechanical dyssynchrony in a porcine model of myocardial infarction by cardiovascular magnetic resonance. J Cardiovasc Magn Reson 2012, 14:1.

22. Fischer SE, Wickline SA, Lorenz CH: Novel real-time R-wave detection algorithm based on the vectorcardiogram for accurate gated magnetic resonance acquisitions. Magn Reson Med 1999, 42:361-370.

23. Look DC, Locker DR: Time saving in measurement of NMR and EPR relaxation times. Rev Sci Instrum 1970, 41:250-251.

24. Kellman P, Arai AE, McVeigh ER, Aletras AH: Phase-sensitive inversion recovery for detecting myocardial infarction using gadolinium-delayed hyperenhancement†. Magn Reson Med 2002, 47:372-383.

25. Fischer SE, McKinnon GC, Maier SE, Boesiger P: Improved myocardial tagging contrast. Magn Reson Med 1993, 30:191-200.

26. Osman NF, McVeigh ER, Prince $\mathrm{JL}$ : Imaging heart motion using harmonic phase MRI. Med Imaging, IEEE Trans 2000, 19:186-202.

27. Abd-Elmoniem KZ, Stuber M, Prince JL: Direct three-dimensional myocardial strain tensor quantification and tracking using zHARP. Med Image Anal 2008, 12:778-786.

28. Amado LC, Gerber BL, Gupta SN, Rettmann DW, Szarf G, Schock R, Nasir K, Kraitchman DL, Lima JA: Accurate and objective infarct sizing by contrast-enhanced magnetic resonance imaging in a canine myocardial infarction model. J Am Coll Cardiol 2004, 44:2383-2389.

29. Burke AP, Virmani R: Pathophysiology of acute myocardial infarction. Med Clin North Am 2007, 91:553-572. ix.

30. Arun KS, Huang TS, Blostein SD: Least-squares fitting of Two 3-D point sets. Pattern Anal Mach Intell, IEEE Trans 1987, PAMI-9:698-700.

31. Cerqueira MD, Weissman NJ, Dilsizian V, Jacobs AK, Kaul S, Laskey WK, Pennell DJ, Rumberger JA, Ryan T, Verani MS: Standardized myocardial segmentation and nomenclature for tomographic imaging of the heart: a statement for healthcare professionals from the cardiac imaging committee of the council on clinical cardiology of the American Heart Association. Circulation 2002, 105:539-542.

32. Moore CC, Lugo-Olivieri CH, McVeigh ER, Zerhouni EA: Three-dimensional Systolic Strain Patterns in the Normal Human Left Ventricle: Characterization with Tagged MR Imaging1. Radiology 2000, 214:453-466.

33. DeLong ER, DeLong DM, Clarke-Pearson DL: Comparing the areas under two or more correlated receiver operating characteristic curves: a nonparametric approach. Biometrics 1988, 44:837-845.

34. Sasano T, Kelemen K, Greener ID, Donahue JK: Ventricular tachycardia from the healed myocardial infarction scar: validation of an animal model and utility of gene therapy. Hear Rhythm 2009, 6:S91-S97.

35. Kim RJ, Wu E, Rafael A, Chen E-L, Parker MA, Simonetti O, Klocke FJ, Bonow RO, Judd RM: The Use of contrast-enhanced magnetic resonance imaging to identify reversible myocardial dysfunction. New Engl J Med 2000, 343:14451453. 
36. Marsan NA, Westenberg JJM, Ypenburg C, van Bommel RJ, Roes S, Delgado V, Tops LF, van der Geest RJ, Boersma E, de Roos A, et al.: Magnetic resonance imaging and response to cardiac resynchronization therapy: relative merits of left ventricular dyssynchrony and scar tissue. Eur Heart J 2009, 30:2360-2367.

37. Ashikaga H, Sasano T, Dong J, Zviman MM, Evers R, Hopenfeld B, Castro V, Helm RH, Dickfeld T, Nazarian S, et al.: Magnetic resonance-based anatomical analysis of scar-related ventricular tachycardia. Circul Res 2007, 101:939-947.

doi:10.1186/1532-429X-14-85

Cite this article as: Soleimanifard et al:: Three-dimensional regional strain analysis in porcine myocardial infarction: a 3T magnetic resonance tagging study. Journal of Cardiovascular Magnetic Resonance 2012 14:85.

\section{Submit your next manuscript to BioMed Central and take full advantage of:}

- Convenient online submission

- Thorough peer review

- No space constraints or color figure charges

- Immediate publication on acceptance

- Inclusion in PubMed, CAS, Scopus and Google Scholar

- Research which is freely available for redistribution 